\title{
A PERIODICALLY-SWITCHED ODE MODEL FOR N-BUNCH BEAMLOADING IN A STORAGE RING*
}

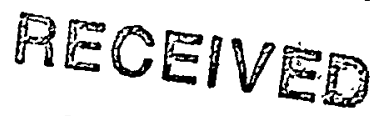

SEP 2 2 C. Schwartz, A. Nassiri

Absr $@$ S S.T.Th

formulation of the coupled cavity/longitudinal-bunch ODEs is derived. Assuming linearity, a model of the form $\dot{x}(t)=A(t) x(t)+B(t) u(t)$ arises, where $A(t)$ and $B(t)$ are piecewise constant, and periodic with the revolution period $T_{0}$. Such models, known in the control community as (periodic) switched systems, have known (in)stability criteria and control theoretic properties, which can be useful in the analysis and control of multiple bunch beamloading.

\section{BASEBAND FORMULATION}

The term beamloading is to imply here the dynamical interaction between a given cavity resonance and the $N$ bunches' longitudinal dynamics. Resonance implies bandlimitedness (BL), and a standard tool for the analysis of bandlimited signals and systems (ODEs) is the IQ formalism [1]. The formalism has been applied to beamloading, especially w.r.t. the cavity ODE in [2], [3].

In the way of review and to establish notation: The Fourier transform of the resonance $Z(j \omega)$, of order $M$ in $j \omega$, is assumed to be (effectively) zero for $\omega$ outside its band. Denote the positive part of the band by $\Omega$. Then using some carrier frequency $\omega_{c} \in \Omega$, the impulse response kernel of $Z(j \omega)$ is

$$
z(t)=z_{I}(t) \cos \omega_{c} t-z_{Q}(t) \sin \omega_{c} t .
$$

The utility of the IQ formalism lies in the fact that we need only consider the complex envelope, defined as $\bar{z}(t)=$ $z_{I}(t)+j z_{Q}(t)$, whose Fourier transform $\tilde{Z}(j \omega)$ is also of order $M$ in $j \omega$. In particular, the cavity output signal $v(t)$ to an (AM/PM) sinusoid $f(t)$ is obtained via $\tilde{v}(t)=\tilde{f}(t) * \tilde{z}(t)$

\subsection{Bunch Train Signal}

Use of the IQ formalism presupposes AM/PM signals of the form (1). It is now shown that the beam current, modeled here as an impulse train, is seen by the resonance approximately as an AM/PM signal about the carrier $\omega_{c}$.

The width of $\Omega$ determines the minimum number of bunches that need be considered in a time domain analysis; arbitrary gaps in the beam current may make this determination difficult. Here, the number of representative bunches $N$ is assumed known, chosen through modal analysis or made safely large.

\footnotetext{
- Work supported by U.S. Department of Energy, Office of Basic Energy Sciences, under Contract No. W-31-109-ENG-38.
}

Define $N_{o}$ as the number of bunch current "segments": each $n$th beam current segment is of duration $T_{b}=T_{o} / N_{o}$ and has a charge $q_{n}, n=1, \ldots, N_{o} . q_{n}$ may be identically zero if and only if the segment represents a gap; if there are no gaps $N=N_{0}$. Henceforth the word bunch shall mean bunch segment.

Let $\tau_{n, p}$ denote the $n$th bunch's deviation in arrival time at the cavity from the nominal, for the $p$ th arrival. Of course if $q_{n}=0$ then $\tau_{n}$. is devoid of physical meaning; otherwise it is governed by:the synchrotron ODE. However, the cavity sees the beam cưrrent as a signal, and that is the perpective of this section.

The time-infinite beam current is written, using Wilson's phasor convention [4], as

$$
\cdot i(t)=-\sum_{n=1}^{N_{o}} q_{n} \sum_{p=-\infty}^{\infty} \delta\left(t-\left[p+\frac{n-1}{N_{o}}\right] T_{o}-\tau_{n, p}\right)
$$

but, as proven in section 1.2, the following Proposition applies:

Proposition The beam current (2) is seen by an $\Omega-B L$ resonance approximately as

$$
i(t) \approx-q(t) \frac{2}{T_{b}}\left[\cos \omega_{c} t+\omega_{c} \tau(t) \sin \omega_{c} t\right] .
$$

In (3), $q(t)=q\left(t+T_{0}\right)$ is a continous-time interpolation (CTI) of $q_{n}, n=1, \ldots, N_{o}$, and $\tau(t)$ is a CTI of $\tau_{n, p}, \forall n, p$, as depicted in Figures 1-2 and defined in the next section. Note that $\tau(t)$ is of use only in discussing the beam current as a signal; when addressing the system aspect (section 1.3 and on), $\tau(t)$ will be abandoned.

\subsection{Proof of the Proposition}

The Proposition is proved in three steps: interpolation, Taylor series.approximation, and application of some Fourier properties.

Interpolation [1] The signals $q(t)$ and $\tau(t)$ are formally constructed via the interpolation kernel $S_{T_{2}}(t)=u(t+$ $\left.T_{1} / 2\right)-u\left(t-T_{1} / 2\right)$, where $T_{1}$ is some period, and $u(t)=1$ for $t \geq 0$, and is zero otherwise. Define $\bar{q}_{k}=q_{-1+k \bmod N_{0}}$. Then formally,

$$
\begin{aligned}
q(t) & =\sum_{k=-\infty}^{\infty} \bar{q}_{k} S_{T_{b}}\left(t-k T_{b}\right) \\
\tau(t) & =\sum_{p=-\infty}^{\infty} \sum_{n=1}^{N_{o}} \tau_{n, p} S_{T_{b}}\left(t-\frac{n-1}{N_{o}}-p T_{b}\right) .
\end{aligned}
$$




\section{DISCLAIMER}

This report was prepared as an account of work sponsored by an agency of the United States Government. Neither the United States Government nor any agency thereof, nor any of their employees, make any warranty, express or implied, or assumes any legal liability or responsibility for the accuracy, completeness, or usefulness of any information, apparatus, product, or process disclosed, or represents that its use would not infringe privately owned rights. Reference herein to any specific commercial product, process, or service by trade name, trademark, manufacturer, or otherwise does not necessarily constitute or imply its endorsement, recommendation, or favoring by the United States Government or any agency thereof. The views and opinions of authors expressed herein do not necessarily state or reflect those of the United States Government or any agency thereof. 


\section{DISCLAIMER}

Portions of this document may be illegible in electronic image products. Images are produced from the best available original document. 


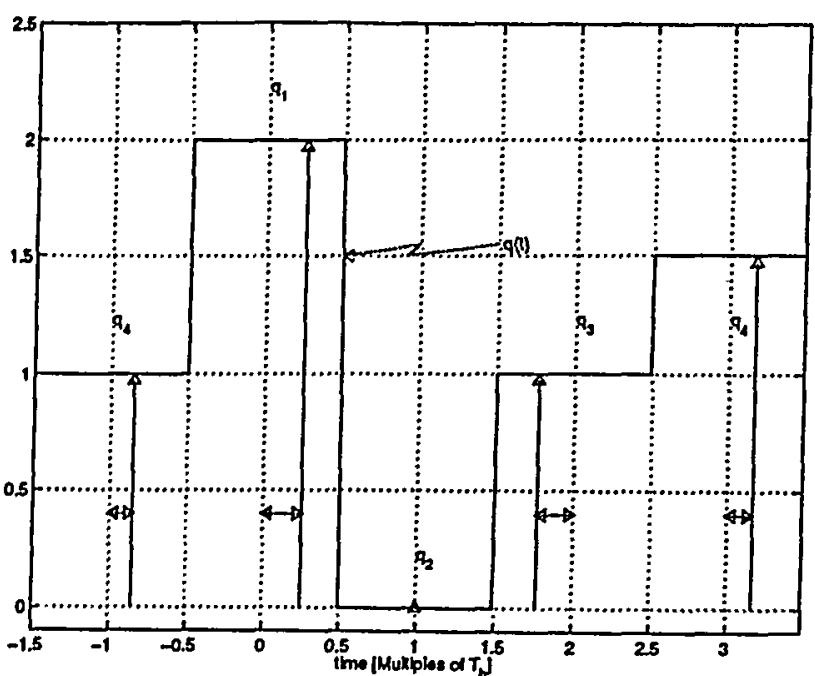

Figure 1: $i(t)$ and $q(t)$ for a case where $N_{0}=4, N=3$ (ordinate units are arbitrary). $q(t)$ is the "envelope" of $i(t)$. The horizontal arrows indicate arrival time deviations and are interpolated sequentially into $\tau(t)$.

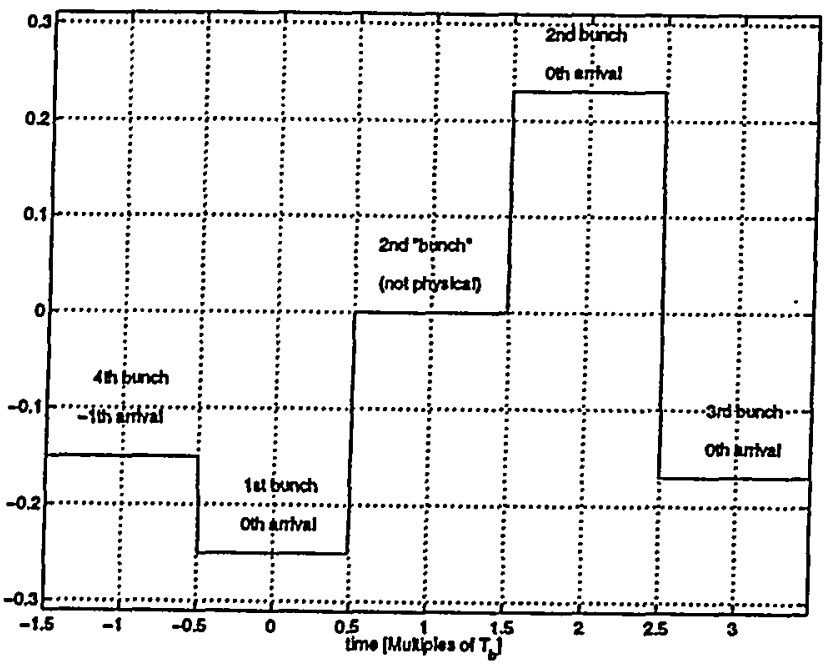

Figure 2: $\tau(t)$ as interpolated from the $\tau_{n, p}$ of $i(t)$ of Figure 1.

Since $y(t) \delta(t-a)=y(a) \delta(t-a),(2)$ can be rewritten as

$$
i(t)=-q(t) \sum_{k=-\infty}^{\infty} \delta\left(t-k T_{b}-\tau(t)\right) .
$$

Taylor Series Approximation Assuming that $|\tau(t)|$ of (6) is small for all $t$, then the bilinear (first two) terms of the signal's Taylor series expansion approximate the signal [5]. Hence, the RHS of (6) can be rewritten as

$$
i(t) \approx-q(t) \sum_{k=-\infty}^{\infty}\left[\delta\left(t-k^{\prime} I_{b}\right)-\tau(t) \dot{\delta}\left(t-k T_{b}\right)\right] .
$$

Fourier Analysis Via Fourier series it is known that $\sum \delta\left(t-n T_{1}\right)=2 / T_{1} \sum \cos n \omega_{1} t$, where the sum is for $n$ over the set of all integers. Applying the transform identity $\dot{y}(t) \leftrightarrow j \omega Y(\omega)$ yields that $\sum \dot{\delta}\left(t-n T_{1}\right)=$ $-2 \omega_{1} / T_{1} \sum n \sin n \omega_{1} t$. Thus the RHS of (7) can be written as

$$
q(t) \frac{2}{T_{b}}\left[\sum_{p=0}^{\infty} \cos p \omega_{b} t+\tau(t) \omega_{b} \sum_{p=0}^{\infty} p \sin p \omega_{b} t\right] .
$$

Finally, aside from the considerations mentioned in section 1.1 regarding the choice of $N$ and hence $N_{o}$, here a further imposition on $N_{o}$ is introduced: $N_{o}$ is chosen s.t. $k_{o} \omega_{b} \in \Omega$ for one and only one integer $k_{o}$. Choose the carrier frequency according to $\omega_{c}=k_{0} \omega_{b}$. Thus by design, no harmonics of $\omega_{b}$ fall in the band $\Omega$, and it can be shown (e.g., via convolution) that the $\Omega$-BL resonance sees only the harmonic $p=k_{0}$ of (8), concluding the Proof.

Remark The derivation of (3) does not impose any assumptions on the bunch longitudinal motion other than it is of small amplitude, cf. the traditional derivations using modal analysis ànd Bessel functions [6]. In addition, it allows for gaps in the bunch train.

\subsection{Beamloading ODEs}

It is convenient henceforth to use deviation from nominal values for all variables. Then the cavity portion of the overall beamloading system at baseband can be written as

$$
\begin{aligned}
\widetilde{\Delta v}(t) & =\tilde{z}(t) *\left[\Delta i_{I}(t)+j \Delta i_{Q}(t)\right] \\
& =\tilde{z}(t) * 2\left[\frac{q(t)}{T_{b}}-I_{o}+j \frac{\omega_{c} q(t) \tau(t)}{T_{b}}\right] \\
& \equiv \Delta v_{I I}-\Delta v_{Q Q}+j\left[\Delta v_{I Q}+\Delta v_{Q I}\right]
\end{aligned}
$$

where $I_{0}$ is the DC beam current.

From the perspective of the cavity resonance $\tau(t)$ is a signal, but in truth it is determined over time by $N$ synchrotron ODEs. These are incorporated in the overall system by now abandoning the variable $\tau(t)$, and instead working with the new continuous state variables $\tau_{m}(t), m=1, \ldots, N$, which represent each $m$ th bunch's arrival time deviation from nominal. The relationship between $\tau_{m}(t)$ and $(9)$ will be evident in the following development.

From the perspective of the $m$ th bunch, the cavity voltage represents a forcing function that is nonzero only during the time intervals $T_{m}$, during which the bunch couples to, i.e., passes through, the cavity. For example, bunch No. 1 of Figure 1 passes through the cavity during the intervals

$$
\mathrm{T}_{1}=\bigcup_{p=-\infty}^{\infty}\left[p T_{o}-\frac{T_{b}}{2}, p T_{o}+\frac{T_{b}}{2}\right) .
$$

Otherwise, i.e., $\forall t \in \mathbf{T}_{m}^{c}$, where.$^{c}$ denotes set complement, the bunch is not coupled to the cavity. The set of all time intervals that correspond to gaps is $\mathbf{T}_{o}=n \mathbf{T}_{m}^{c}$.

Defining $\phi_{m}(t)=\omega_{c} \tau_{m}(t)$, the linearized synchrotron ODE can thus be written as

$$
\ddot{\phi}_{m}+2 \alpha \dot{\phi}_{m}+\omega_{s}^{2} \phi_{m}=\left\{\begin{array}{ll}
f_{c}(t) & t \in T_{m} \\
0 & t \in T_{m}^{c}
\end{array},\right.
$$


where $\alpha$ is the inverse dmaping time, and $\omega_{z}$ is the synchrotron frequency. It can be shown that given linearization, the RHS forcing function is given by $f_{c}(t)=$ $\frac{\omega_{c}^{2}}{V_{c} \sin \phi_{e}}\left[\Delta v_{I I}(t)-\Delta v_{Q Q}(t)\right]$, where $V_{c}$ is the peak cavity voltage, and $\phi_{\delta}$ is the synchronous phase.

Remark $\Delta v_{I I}(t)$ is a function of $q(t)$, and hence is not a state but a stationary forcing function, not governed by an ODE. Therefore it does not have any bearing on (linearized) stability, see e.g., [7], Theorem 12.6. Thus, the quadrature impulse response of the cavity resonance $z_{Q}(t)$ and not $z_{I}(t)$ determines linearized beamloading stability, aside from the beam ODEs. Compare the argument proposed in [3] to prove this. Note also that the result is particularly transparent here after having used the phasor reference plane of [4].

\section{SWITCHED SYSTEM FORMULATION}

By merging the cavity (9) and beam (11) system formulations developed above, one arrives at a single ODE of the form $\dot{x}(t)=A(t) x(t)+B(t) \Delta v_{I I}(t)$ : The $M$ cavity states $\Delta v_{Q Q}^{M-1}(t), \ldots, \Delta v_{Q Q}(t)$ and the two bunch states $\phi_{m}^{\prime}(t), \phi_{m}(t)$ for each of the $N$ bunches are ordered in the column vector $x$, where the superscript now denotes derivative order. Thus $x$ has $2 N+M$ elements. The ODE coeffcients are corresponding elements of $A(t)$ and $B(t)$. More specifically, the resulting overall system is of the form

$$
\dot{x}(t)=\left\{\begin{array}{ll}
A_{m} x(t)+B_{m} \Delta v_{I I}(t) & t \in \mathbf{T}_{m} \\
A_{o} x(t) & t \in \mathbf{T}_{o}
\end{array} .\right.
$$

The top part of the RHS conveys that while the $m$ th bunch passes through the cavity, the beam current [quadrature modulated by the state $\left.\phi_{m}(t)\right]$ perturbs the cavity. Meanwhile the bunch is perturbed by the cavity state $\Delta v_{Q Q}(t)$, and by the forcing function $\Delta v_{I I}(t)$. During the periods corresponding to gaps (bottom of RHS), the cavity and bunches are uncoupled. Thus, $A_{o}$ is a $(N+M)$ by $(N+M)$ block diagonal matrix, and $A_{m}$ contains the same block diagonal elements as well as off diagonal coupling terms. $B_{m}$ is $(N+M)$ by 1 , and contains only a single nonzero element.

\section{APPLICATIONS}

Given an initial condition $x_{o}$, say at $t=0$, then (12) represents an initial value problem, $\dot{x}(t)=A(t) x(t), x(0)=$ $x_{o}$. Two properties of the system readily lend themselves to application of ODE system theory, see e.g., [7]. First, $A(t)$ is piecewise constant, or a switched system, which means that the state transition matrix $\Phi(t, 0)$ for any $t$ can be computed as the product of matrix exponentials. For example, in the case of Figure 1, starting at $t=0$, the state at $3.5 T_{b}$ is given by $e^{T_{b} A_{s}} e^{T_{b} A_{2}} e^{T_{b} A_{o}} e^{0.5 T_{b} A_{1}} x_{o}$. Thus, using the state transition matrix, the state $x(t)$ for any $t$ can be computed, see [8].

Second, since $A(t)$ is $T_{o}$ periodic, Floquet Theory can be applied to assess (in)stability:
Theorem [Floquet] The system (12) is stable (unstable) if and only if the magnitudes of the eigenvalues of $\Phi\left(T_{0}, 0\right)$ are s.t. all are less than unity (at least one is greater than unity).

A particularly useful application of this criterion is the identification of the cavity higher mode(s) that cause coupled bunch instabilities in a partially filled storage ring [9]: For each cavity mode, a new $\omega_{c}$ is determined, and then the eigenvalues of the corresponding $\Phi\left(T_{o}, 0\right)$ are checked for stability.

A final application (but orginally the motivating application) is beamloading control. The authors of [10] note that the now classic optimal state space control theory does not readily apply to multiple bunch beamloading. The switched system formulation, along with some recent control-theoretic results relating to the control of such systems [11], are therefore of particular interest and are currently under study.

\section{ACKNOWLEDGEMENTS}

The authors are most grateful to Dr. K. Harkay for her generous and able assistance with physics issues. Dr. A. H. Haddad, Dever Professor in ECE at Northwestern University, has also greatly assisted in his capacity as Doctoral Advisor to C.S. Finally, the financial support of the Accelerator Systems Division of APS is greatfully acknowledged.

\section{REFERENCES}

[1] A. Papoulis, Signal Analysis, McGraw Hill, (1977).

[2] S. J. Jachim and B. R. Cheo, "Dynamic Interactions Between RF Sources and LINAC Cavities with Beam Loading," IEEE Trans. Electron Devices, October 1991.

[3] S.Y. Zhang and W.T. Weng, "A New Formulation of Longitudinal Coherent Instabilities," Proc. 1993 PAC, (1993).

[4] P.B. Wilson, "High Energy Electron Linacs: Applications to Storage Ring RF Systems and Linear Colliders," SLAC2884, (1982).

[5] A. Papoulis, "Error Analysis in Sampling Theory," Proc. IEEE, 54(7), July 1966.

[6] A. Hofmann, "Beam Instabilites," CAS-CERN Accelerator School Fifth Advanced Physics Course, CERN (1993).

[7] W. J. Rugh, Linear System Theory, Prentice Hall, (1996).

[8] K. L. F. Bane et al., "Compensating the Unequal Bunch Spacing in the NLC Damping Rings," Proc. Fifth EPAC, (1996).

[9] K. Harkay et al., "Compensation of Longitudinal CoupledBunch Instability in the APS Storage Ring," Proc. PAC-97, pp. 1575, (1997).

[10] D. Boussard and E. Onillon, "Application of the methods of optimum control theory to the if system of a circular accelerator," CERN SL 93-09 RFS, 1993.

[11] J. Ezzine and A. H. Haddad, "Controllability and Observability of Hybrid Systems," Intl. J. of Control, 49(6), pp. 2045-2055, June 1989. 